\title{
Prediction and compensation of machining geometric errors of five- axis machining centers with kinematic errors
}

\section{$\operatorname{AUTHOR}(\mathrm{S}):$}

Uddin, M. Sharif; Ibaraki, Soichi; Matsubara, Atsushi; Matsushita, Tetsuya

\section{CITATION:}

Uddin, M. Sharif...[et al]. Prediction and compensation of machining geometric errors of five-axis machining centers with kinematic errors. Precision Engineering 2009, 33(2): 194201

\section{ISSUE DATE:}

2009-04

URL:

http://hdl.handle.net/2433/123362

\section{RIGHT:}

Copyright @ 2008 Elsevier; この論文は出版社版でありません。引用の 際には出版社版をご確認ご利用ください。; This is not the published version. Please cite only the published version. 


\title{
Prediction and Compensation of Machining Geometric Errors of Five-axis Machining Centers with Kinematic Errors
}

\author{
M. Sharif Uddin ${ }^{1}$, Soichi Ibaraki ${ }^{1 *}$, Atsushi Matsubara ${ }^{1}$, Tetsuya Matsushita ${ }^{2}$ \\ ${ }^{1}$ Dept. of Micro Engineering, Graduate School of Engineering, Kyoto University \\ Yoshida-honmachi, Sakyo-ku, Kyoto 606-8501, Japan \\ *Email: ibraki@prec.kyoto-u.ac.jp \\ ${ }^{2}$ Okuma Corporation, Aichi 480-0193, Japan,
}

\begin{abstract}
:
Kinematic errors due to geometric inaccuracies in five-axis machining centers cause deviations in tool positions and orientation from commanded values, which consequently affect geometric accuracy of the machined surface. As is well known in the machine tool industry, machining of a cone frustum as specified in NAS979 standard is a widely accepted final perforance test for five-axis machining centers. A critical issue with this machining test is, however, that the influence of the machine's error sources on the geometric accuracy of the machined cone frustum is not fully understood by machine tool builders and thus it is difficult to find causes of machining errors. To address this issue, this paper presents a simulator of machining geometric errors in five axis machining by considering the effect of kinematic errors on the three-dimensional interference of the tool and the workpiece. Kinematic errors of a five-axis machining center with tilting rotary table type are first identified by a DBB method. Using an error model of the machining center with identified kinematic errors and considering location and geometry of the workpiece, machining geometric error with respect to the nominal geometry of the workpiece is predicted and evaluated. In an aim to improve geometric accuracy of the machined surface, an error compensation for tool position and orientation is also presented. Finally, as an example, the machining of a cone frustum by using a straight end mill, as described in the standard NAS979, is considered in case studies to experimentally verify the prediction and the compensation of machining geometric errors in five axis machining.
\end{abstract}

Keywords: five -axis machining center, kinematic errors, DBB method, machining geometric errors, the cone frustum workpiece, Error compensation

\footnotetext{
${ }^{*}$ The corresponding author
} 


\section{Introduction}

With a tremendously increasing need for machined components with geometric complexity and high dimensional accuracy, recently five-axis machining centers are extensively used in various manufacturing. Because of its versatile functionalities, a five-axis machining center offers notable benefits that include increased material removal rate, enhanced surface accuracy, and reduced effective machining time. However, it is well known that kinematic errors due to geometric inaccuracies of structural components in a five-axis machining center can cause significant errors on the tool position and orientation with respect to workpiece, hence leading to an inaccuracy of machined surface in actual cutting operation.

As a standard describing the inspection of motion accuracy of a five-axis machine tool, ISO 10791 [1] defines measurement schemes to evaluate kinematic errors using displacement sensors and artifacts for five-axis machine configurations having a universal head. For five-axis machine configurations having a tilting rotary table, ISO 230-7 [2] was recently published in 2005 developing a measurement scheme of kinematic errors of rotary axes. Although it is crucial to evaluate each kinematic error by using such a measurement, typical machine tool users concern more the accuracy of a five-axis machine when it performs actual machining. NAS 979 [3] describes the evaluation of the machining accuracy of a five-axis machine by the machining of a cone frustum. Since it is only standard describing a machining test by five-axis machines well known in the machine tool industry, a machining test of a cone frustum is widely accepted to many machine tool builders as a final performance test for five-axis machines. A critical issue with this machining test is, however, that the influence of the machine's error sources on the geometric accuracy of the machined cone frustum is not fully understood by machine tool builders. Therefore, it is generally very difficult for machine tool builders to find causes of geometric errors of the machined cone frustum. Unlike conventional 3-axis machining centers, it is difficult to intuitively understand the effect of the machine's motions errors on the machining accuracy in five-axis machines. Another critical issue with NAS 979 is that it does not refer to the location of the workpiece to be machined. On five-axis machines, the influence of kinematic errors of each axis on the overall positioning error of the tool with respect to the workpiece may significantly differ depending on the position of each axis.

The effect of kinematic errors on motion accuracy in various types of five-axis machines has been investigated by constructing their kinematic model [4-7]. There has been also numerous research works reported in the literature on the identification of kinematic errors based on the measurement of the machine's motion error. The telescoping double ball bar (DBB) measuring device has been applied to identify kinematic errors on five-axis machines [8-13]. Lei and Hsu [14] applied a 3D probe ball device to identify some of kinematic errors. Bringmann and Knapp [15] 
showed the 'R-Test' measurement device, where the three-dimensional displacement of the tool center is measured by using a sphere attached to the spindle tip, and four displacement sensors pointed to the center of the sphere. In all these approaches, kinematic errors are identified from the measured positioning error of the machine based on its kinematic model.

While research works reviewed above have focused on the measurement of kinematic errors, no or little work is available in literature, focusing on the explicit analysis on the effect of kinematic errors in five-axis machining centers on the machining geometric accuracy. In particular, it is of a practical importance to exploit the effect of kinematic errors on the geometric error of a cone frustum machined by using a five-axis machine, as a basis to establish a diagnosis methodology of errors sources from machining results or to implement a compensation scheme of machining errors. Although some latest papers $[16,17]$ discuss the effect of kinematic errors on the machining error of a cone frustum, they only consider the displacement at the tool tip in the simulation, and thus do not consider the three-dimensional interference of the tool and the workpiece.

The present research work proposes a simulator of machining errors in five-axis machining by considering the effect of kinematic errors on the three-dimensional interference of the tool and the workpiece. Assuming that kinematic errors of a five-axis machine are identified by applying one of the approaches reviewed above, the simulator presented in this paper predicts machining geometric errors with respect to the nominal geometry of the workpiece. Such a simulator is crucial to quantitatively evaluate the significance of each kinematic error on the machine's overall machining accuracy. In order to enhance the geometric accuracy on machined workpiece surface, an error compensation for tool position and orientation is also presented. Finally, as an example, the machining of the cone frustum by using a straight end mill, as described in the standard NAS979, is considered in case studies to experimentally verify the prediction and compensation of machining errors in five-axis machining.

\section{Kinematic modeling of five-axis machining centers}

\subsection{Configurations of five-axis machining centers}

Generally a five-axis machining center has three linear axes and two controllable rotary axes. There are different types of five-axis machining centers currently available in the machine tool manufacturing market. Beside of some with special configuration, five-axis machining centers with three types of configuration are very commonly used in the industries [12]. The first is a universal head type with two rotary axes. The second is a tilting rotary table type with two rotary axes. And the third is a kind of combination of first and second types with a swivel head and a rotary table. 
In this study, a five-axis machining center with the tilting rotary table type, or ZX/YAC configuration [18] is considered as the target. Figure 1 shows its basic configuration. As can be seen in Fig.1, the machine contains three linear-axis drives $(\mathbf{X}, \mathbf{Y}, \mathbf{Z})$ for generating linear motions in $X$, $Y$, and $Z$ directions and two rotary-axis drives (A,C) for generating rotary motions on the tiltingrotary table about $X$ and $Z$ axes respectively. Although this paper only considers this most popular configuration of five-axis machine tools, it must be emphasized that simulation and compensation schemes of machining geometric errors presented in this paper can be straightforwardly extended to any configurations of five-axis machines.

\subsection{Definitions of kinematic errors}

Inasaki et al. [19] have pointed out that eleven kinematic errors are sufficient to define the relative location and orientation of all the linear and rotary axes in the configuration shown in Fig. 1. Namely, eight kinematic errors $\left(\alpha_{A Y}, \beta_{A Y}, \gamma_{A Y}, \beta_{C A}, \delta x_{A Y}, \delta y_{A Y}, \delta z_{A Y}, \delta y_{C A}\right)$ are associated with rotary axes and three kinematic errors $\left(\gamma_{Y X}, \alpha_{Z Y}, \beta_{Z X}\right)$ are associated with linear axes. The definitions of these kinematic errors are briefly described as follows [19]:

$\alpha_{A Y}$ is the angular error of $\mathrm{A}$-axis with respect to $\mathrm{Y}$-axis about $\mathrm{X}$-axis. Similarly, $\beta_{A Y}$ and $\gamma_{A Y}$ are the angular errors of $\mathrm{A}$-axis about $\mathrm{Y}$ and $\mathrm{Z}$-axes respectively. $\beta_{C A}$ is the angular error of $\mathrm{C}$-axis with respect to $\mathrm{A}$-axis about $\mathrm{X}$-axis. $\delta x_{A Y}, \delta y_{A Y}$, and $\delta z_{A Y}$ are linear shifts of A-axis with respect to $\mathrm{Y}$-axis (or the origin of machine, $\mathrm{XYZ}$ ) in $\mathrm{X}, \mathrm{Y}$ and $\mathrm{Z}$ directions respectively. $\delta y_{C A}$ is the linear shift of $\mathrm{C}$-axis with respect to A-axis in Y-direction. On the other hand, $\gamma_{Y X}, \alpha_{Z Y}$, and $\beta_{Z X}$ are defined as the orthogonality or squareness errors of three linear axes (XYZ) on X-Y, Y-Z, and Z-X planes respectively. Note that these eleven kinematic errors will be considered in error modeling of the machine tool and then, effects of them on machining accuracy will be evaluated as well.

\subsection{Kinematic modeling of five-axis machining center with kinematic errors}

The kinematic model of the machine configuration in Fig. 1 is given in details in [12]. In this paper, the objective to employ the kinematic model of the machining center is to calculate the tool center location relative to the workpiece under the existence of kinematic errors. This section briefly reviews this calculation based on the kinematic model. Coordinate systems for the machine configuration shown in Fig. 1 are illustrated in Fig. 2.

First, the location of the tool center in the reference frame is calculated with kinematic errors. Here, the reference frame $\left(\mathrm{X}_{\mathrm{F}}-\mathrm{Y}_{\mathrm{F}}-\mathrm{Z}_{\mathrm{F}}\right)$ is the coordinate system fixed to the machine frame or bed as

shown in Fig. 2. When the command position of the tool center is given by $\left(\hat{X}_{c}, \hat{Y}_{c}, \hat{Z}_{c}\right)$, the actual tool center location in the reference frame under the influence of the machine's kinematic 
errors, ${ }^{r} p_{t} \in \mathfrak{R}^{4}$, is given as follows. First three elements of ${ }^{r} p_{t}$ represent $\mathrm{X}, \mathrm{Y}$ and $\mathrm{Z}$ coordinates and its fourth element is one. Note that the left-side superscript $r$ denotes the vector defined in the reference frame.

${ }^{r} p_{t}={ }^{r} T_{z}{ }^{z} T_{x} p_{t}^{0}$

where $p_{t}^{0}=\left[\begin{array}{llll}0 & 0 & 0 & 1\end{array}\right]^{T} .{ }^{r} T_{z} \in \mathfrak{R}^{4 \times 4}$ is a homogeneous transformation matrix (HTM) [20] representing the motion of the $\mathrm{Z}$ axis in the reference frame. Similarly, ${ }^{z} T_{x} \in \mathfrak{R}^{4 \times 4}$ denotes a HTM representing the motion of the $\mathrm{X}$ axis with respect to the $\mathrm{Z}$ axis. They are respectively given by:

$$
\begin{aligned}
& { }^{r} T_{z}=D^{4}\left(\alpha_{Z Y}\right) D^{5}\left(\beta_{Z X}\right) D^{3}\left(\hat{Z}_{c}\right) \\
& { }^{z} T_{x}=D^{1}\left(\hat{X}_{c}\right)
\end{aligned}
$$

where, $\alpha_{Z Y}$ and $\beta_{Z X}$ are kinematic errors defined in Section 2.2. $D^{1-6}(*) \in \mathfrak{R}^{4 \times 4}$ represent the HTMs, where $D^{1}(x), D^{2}(y)$, and $D^{3}(z)$ respectively represent the HTM for linear motions in the $\mathrm{X}, \mathrm{Y}$, and Z directions, and $D^{4}(a), D^{5}(b)$, and $D^{6}(c)$ respectively represent the HTM for angular motions about X, $\mathrm{Y}$ and $\mathrm{Z}$ axes.

Then, the transformation from the workpeice frame to the reference frame is considered. Note that the workpiece frame $\left(\mathrm{X}_{\mathrm{w}}-\mathrm{Y}_{\mathrm{w}}-\mathrm{Z}_{\mathrm{w}}\right)$ is attached to the workpiece as shown in Fig. 2. The HTM representing the transformation from the workpiece frame to the reference frame is given by:

$$
{ }^{r} T_{w}={ }^{r} T_{y}{ }^{y} T_{a}{ }^{a} T_{c}
$$

where the HTMs, ${ }^{a} T_{c},{ }^{y} T_{a}$, and ${ }^{r} T_{y}$ are respectively given as:

$$
\begin{aligned}
& { }^{a} T_{c}=D^{2}\left(\delta y_{C A}\right) D^{5}\left(\beta_{C A}\right) D^{6}(\hat{C}) \\
& { }^{y} T_{a}=D^{1}\left(\delta x_{A Y}\right) D^{2}\left(\delta y_{A Y}\right) D^{3}\left(\delta z_{A Y}\right) D^{4}\left(\alpha_{A Y}\right) D^{5}\left(\beta_{A Y}\right) D^{6}\left(\gamma_{A Y}\right) D^{4}(\hat{A}) \\
& { }^{r} T_{y}=D^{6}\left(\gamma_{Y X}\right) D^{2}\left(-\hat{Y}_{c}\right)
\end{aligned}
$$

where, $\alpha_{A Y}, \beta_{A Y}, \gamma_{A Y}, \beta_{C A}, \delta x_{A Y}, \delta y_{A Y}, \delta z_{A Y}, \delta y_{C A}$, and $\gamma_{Y X}$ are kinematic errors as defined in Section 2.2. $\hat{A}$ and $\hat{C}$ are the tool orientation angles about $\mathrm{X}$ and $\mathrm{Z}$ axes respectively represented in the workpiece frame.

Hence, the tool center location in the workpiece frame, ${ }^{w} p_{t} \in \mathfrak{R}^{4}$, can be given as follows. Note that the left-side superscript $w$ denotes the vector defined in the workpiece frame.

$$
{ }^{w} p_{t}=\left({ }^{r} T_{w}\right)^{-1 r} p_{t}
$$




\section{Simulation of five-axis machining accuracy}

\subsection{Machining of cone frustum}

National Aerospace Standard Association specifies a cone frustum as the test workpiece for evaluating contouring accuracy of the multi-axis machining center (NAS979) [3]. In this standard, after machining, the test part is measured to evaluate the circularity error of circumference, the concentricity error of circumferences, and the angular error of side surface, showing an overall contouring performance of the machining center. The circularity error is defined as the difference between maximum and minimum radial errors of circumference of machined cone frustum. On the other hand, according to NAS979 standard, the difference between maximum and minimum radial errors between circumferences of machined cone frustum and pre-bored hole on the cone frustum is the concentricity error.

In this study, a cone frustum as specified in the standard NAS979 is adopted as the test part to evaluate machining accuracy of the machining center with kinematic errors. Hence, the machining simulation on the cone frustum will be carried out. It must be emphasized that the simulation scheme of machining accuracy presented in the following section is not limited to the machining of a cone frustum. However, since the machining of a cone frustum is widely accepted as a practical machining performance test in the industry, this paper only present the evaluation of machining accuracy of a cone frustum as an application example of practical interest.

Figure 3 shows the machining configuration and parameters of the titled cone frustum to be considered in the present simulation study. In Fig. 3, $\phi \mathrm{D}, \theta$, and $\varphi$ are defined as the diameter of the cone frustum machining tool path in the workpiece frame, the taper angle, and the tilt angle of the cone frustum respectively, while $\left(C_{x}, C_{y}, C_{z}\right)$ is the center location of the workpiece.

\subsection{Simulation of five-axis machining error}

By using the kinematic model presented in Section 2.3, for any given commanded tool position and orientation, one can compute the actual tool position and orientation by considering the effect of kinematic errors. For the computation of machining geometric errors, the threedimensional interference of the tool and the workpiece must be computed. Therefore, an error in the tool orientation must be evaluated as well as an error in the tool position.

For the simulation of the workpiece geometry machined by an end mill, many commercial CAM or machining simulation software adopt a discrete modeling of the workpiece using e.g. the Z-map model [21] or the voxel model [22]. In this paper, the objective of applying the machining simulation is to evaluate the geometric accuracy of the machined workpiece along some given reference trajectories. For example, in the machining of a cone frustum, geometric errors of top and 
bottom circular surfaces (including the circularity error of the circumference, the concentricity error, the flatness error, the parallelism error and so on) are of sole interest. Therefore, it is sufficient to construct the discretized model of workpiece geometry only on the region of interest, which makes it easy to minimize the simulation error due to the discretization by reducing the size of each unit model. In the case of a cone frustum, circumferences of top and bottom surfaces are discritized into a set of points, and the interference of the tool and the segment, which starts from each point on circumferences to the direction normal to the frustum surface, is geometrically calculated to compute the geometric error of the machined surface.

First assume that a set of points that defines the nominal geometry of the region of interest, $W_{i} \in \mathfrak{R}^{3}\left(i=1, \ldots, N_{w}\right)$ is given, as well as a set of unit vectors normal to the nominal geometry, $v_{w, i} \in \mathfrak{R}^{3}\left(i=1, \ldots, N_{w}\right)$. In the case of a cone frustum, $W_{i}$ and $v_{w, i}$ can be given as illustrated in Fig. 4. Also assume that the tool is a straight end mill, and is modeled simply as a cylinder of the tool radius, $r$. Suppose that the commanded trajectory of tool tip location in the workpiece frame is given by ${ }^{w} \hat{p}_{j} \in \mathfrak{R}^{3}\left(j=1, \ldots, N_{p}\right)$ and the commanded tool orientation vector in the workpiece frame is given by ${ }^{w} \hat{v}_{j} \in \mathfrak{R}^{3}\left(j=1, \ldots, N_{p}\right)$. The detailed algorithm for the computation of geometric error of the machined surface is illustrated as follows.

(1) For given $\mathrm{j}$, compute the actual tool center position, ${ }^{w} p_{j} \in \mathfrak{R}^{3}$, and the tool orientation vector, ${ }^{w} v_{t, j} \in \mathfrak{R}^{3}$, using the kinematic model presented in Section 2.3 with given kinematic errors.

(2) Define the plane, $\Theta_{j}$, that is parallel to both of the tool orientation vector, ${ }^{w} v_{t, j}$ and the tool motion direction vector, $M_{t, j}={ }^{w} p_{j+1}-{ }^{w} p_{j}$, and is apart by the distance, $r$ from the tool center, ${ }^{w} p_{j}$, as illustrated in Fig. 5. The plane, $\Theta_{j}$, can be represented by the following equation.

$\Theta_{j}=\left\{q \in R^{3}: q={ }^{w} p_{j}+{ }^{w} \xi_{j}+\left(\alpha \cdot v_{t, j}+\beta \cdot M_{t, j}\right)\right\}$

where ${ }^{w} \xi_{j}$ is the vector that perpendicular to both ${ }^{w} v_{t, j}$ and $M_{t, j}$, and has the length of $r$. $\alpha, \beta \in \mathfrak{R}$ are parameters.

(3) Find a set of $W_{i}$ 's that is within some threshold distance from ${ }^{w} p_{j}$. For all these $W_{i}$ 's, compute the intersection point, $q_{i} \in \mathfrak{R}^{3}$, of the line, $W_{i}+\gamma \cdot v_{w, i}$, where $\gamma \in R$ is a parameter, and the plane, $\Theta_{j}$. When the intersection, $q_{i}$, is given in the above equation with $0 \leq \beta \leq 1$, compute the distance, $d_{j, i}$, as follows.

$$
d_{j, i}=\left\|W_{i}-q_{i}\right\| \cdot \operatorname{sign}(\gamma)\left(\text { or } d_{j, i}=\gamma\right)
$$

(4) Make $j=j+1$, and repeat (1) (3) till $j=N_{p}$ 
(5) For all $i$ 's, take $d_{i}=\min _{j=1, \ldots, N_{p}} d_{j, i}$. This gives the actual machined surface at the nominal workpiece point, $W_{i}$, in the direction of $v_{w, i}$.

\section{Error compensation}

When kinematic errors are accurately identified and thus the error in the tool position and orientation can be estimated by using the kinematic model, an error compensation can be applied to reach the ideal position and orientation by modifying the reference trajectory. In the literature, compensation schemes have been presented [23-25]. In this paper, we adopt the following simple error compensation scheme to cancel the effect of kinematic errors.

When the commanded tool position and orientation in the workpiece frame are respectively given by ${ }^{w} \hat{p}_{t}$ and, ${ }^{w} \hat{v}_{t}$, compute the actual tool position and orientation in the workpiece frame, ${ }^{w} p_{t}$ and ${ }^{w} v_{t}$, under the influence of kinematic errors. Then, by simply modifying the commanded tool position and orientation as follows, the effect of kinematic errors can be cancelled, under the assumption that the error induced by kinematic errors is sufficiently small.

The errors in tool position, ${ }^{w} \delta p_{t}$, and orientation, ${ }^{w} \delta v_{t}$, between erroneous and commanded tool path trajectories are respectively computed as:

$$
\begin{aligned}
& { }^{w} \delta p_{t}={ }^{w} p_{t}-{ }^{w} \hat{p}_{t} \\
& { }^{w} \delta v_{t}={ }^{w} v_{t}-{ }^{w} \hat{v}_{t}
\end{aligned}
$$

Then, the compensated tool center position, ${ }^{w} \widetilde{p}_{t}$, and orientation, ${ }^{w} \widetilde{v}_{t}$, in the workpiece frame is calculated by simply canceling the errors in tool position and orientation from those for the nominal tool path trajectory as follows:

$$
\begin{aligned}
& { }^{w} \widetilde{p}_{t}={ }^{w} \hat{p}_{t}-{ }^{w} \delta p_{t} \\
& { }^{w} \widetilde{v}_{t}={ }^{w} \hat{v}_{t}-{ }^{w} \delta v_{t}
\end{aligned}
$$

\section{Case studies}

In order to justify the prediction and compensation of machining geometric errors on a fiveaxis machining center with its kinematic errors, case studies with cutting experiments on the cone frustum workpiece are demonstrated. The machining tests are conducted by using a five-axis machining center with the configuration shown in Fig.1.

\subsection{Identification of kinematic errors}


In this study, a DBB measurement method is applied to identify the kinematic errors considered. DBB tests with specific measurement patterns proposed by Tsutsumi and Saito [12] are carried out on the target five-axis machining center to identify its kinematic errors. This section briefly reviews the identification scheme of kinematic errors proposed in [12] and presents experimental results of DBB measurements. First, squareness errors associated with linear axes, namely, $\gamma_{Y X}, \alpha_{Z Y}$, and $\beta_{Z X}$, can be identified by performing the DBB measurements on XY, YZ, and ZX planes with only linear axes driven.

Then, four DBB tests are conducted by simultaneously controlling three-axis motion where two linear and one rotary axis motions exist, to estimate eight kinematic errors $\left(\alpha_{A Y}, \beta_{A Y}, \gamma_{A Y}, \beta_{C A}\right.$, $\left.\delta x_{A Y}, \delta y_{A Y}, \delta z_{A Y}, \delta y_{C A}\right)$ associated with rotary axes (A,C) in the five-axis machining center. For the DBB measurement pattern 1, the ball bar is set on the work-table in parallel with the center line of the $\mathrm{C}$-axis, and two linear axis motions on $\mathrm{XY}$ plane, and a rotary motion on $\mathrm{C}$-axis are simultaneously controlled as shown in Fig. 6(a). The relationship between the center shift $\left(e_{x 1}\right.$ and $e_{y 1}$ ) of the measured displacement trajectory and the kinematic errors associated with the DBB measurement pattern $1\left(\alpha_{A Y}, \beta_{A Y}\right.$ and $\left.\beta_{C A}\right)$ can be given as follows.

$\left\{\begin{array}{l}e_{x 1}=R_{C 1} \beta_{A Y}+R_{C 1} \beta_{C A} \\ e_{y 1}=-R_{C 1} \alpha_{A Y}\end{array}\right.$

where, $R_{C 1}$ is the distance between the center of the table-side ball and the center line of C-axis in the radial direction of C-axis. Similarly, DBB measurement patterns 2, 3 and 4 are illustrated in Fig. 6(b), 6(c), and 6(d) respectively. When the center shift of the measured displacement trajectory in DBB measurement patterns 2,3 , and 4 is respectively given by $\left(e_{y 2}, e_{z 2}\right),\left(e_{y 3}, e_{z 3}\right)$, and $\left(e_{x 4}, e_{y 4}\right)$, their relationships with kinematic errors are given by:

$$
\begin{aligned}
& \left\{\begin{array}{l}
e_{y 2}=-R_{C 2} \gamma_{A Y}+R_{C 2} \gamma_{Y X} \\
e_{z 2}=R_{C 2} \beta_{A Y}-R_{C 2} \beta_{Z X}
\end{array}\right. \\
& \left\{\begin{array}{l}
e_{y 3}=-\delta y_{A Y} \\
e_{z 3}=-\delta z_{A Y}
\end{array}\right. \\
& \left\{\begin{array}{l}
e_{x 4}=-\delta x_{A Y} \\
e_{y 4}=-\delta y_{A Y}-\delta y_{C A}
\end{array}\right.
\end{aligned}
$$

where, $R_{C 2}$ is the distance between the center of the table-side ball and the center line of A-axis in the radial direction of A-axis. Total eight kinematic errors associated with rotary axes can be identified from measured center shifts $\left(\left(e_{x 1}, e_{y 1}\right), \ldots\left(e_{x 4}, e_{y 4}\right)\right)$ by using Eqs. $(15) \sim(22)$. 
With the above DBB measurement patterns, actual DBB tests are carried out on the machining center of the configuration shown in Fig. 1. During the DBB tests, the nominal length of the ball bar is kept $100 \mathrm{~mm}$. Then, by using the identification equations described above, all the kinematic errors associated with both rotary and linear axes are identified. Table 1 summarizes the identified kinematic errors in the machining center.

\subsection{Prediction of machining errors}

Machining errors simulation and, then cutting experiments of a cone frustum are conducted with three different conditions on the location and the geometry of the workpiece. The machining conditions (Condition I, II, and III) used in both simulation and experiments are summarized in Table 2. During cutting tests in all the cases, a sintered carbide straight end mill of the radius $10 \mathrm{~mm}$ is used. The workpiece material is the aluminum alloy. The side surface of the cone frustum is finished by a single path under the following cutting conditions: the feedrate of $1000 \mathrm{~mm} / \mathrm{min}$, the spindle speed of $5000 \mathrm{~min}^{-1}$ (in Condition I) and $5500 \mathrm{~min}^{-1}$ (in Condition II and III) and the radial depth of cut of $0.01 \mathrm{~mm}$. After the finishing, surface measurements taken by a roundness measuring system (Talycenta1000 by Taylor Hobson, Ltd.) are carried out to measure machining errors on bottom and top surfaces of the machined cone frustum workpiece surface.

Figures 7, 8, and 9 show simulated and measured machining error trajectories with respect to the nominal trajectory for the bottom and top surfaces of the machined cone frustum workpiece. The errors are magnified by 1000 times on both top and bottom surface trajectories. The simulated error trajectories are computed by using the simulator presented in Section 3.2 and identified kinematic errors shown in Table 1. From the figures, we can observe in all the conditions that rough geometries of measured and simulated machining errors trajectories well agree, verifying the effectiveness of the prediction of machining errors.

A comparison of the circularity error and the concentricity error obtained from the simulated and measured machining error trajectories of the machined cone frustum under Condition I, II, and III is summarized in Table 3. It is seen that circularity errors of measured trajectories become larger on both top and bottom surfaces in the order of Condition I-II-III. The tendency in simulated trajectories well matches with this. As is observed in Figs. 7-9, rough geometries of simulated trajectories also well match with measured ones. However, there are still differences in terms of circularity and concentricity errors between simulated and measured trajectories of the machined cone frustum. This could be mostly attributable to higher frequency vibrations observed in measured trajectories, which are not included in the kinematic model used in the present simulation. Simulation errors may be also attributable to other causes, such as static or dynamic motion errors 
of linear or rotary axes, or the tool vibration due to the cutting force. The inclusion of these possible error sources into simulation will be left for our future research.

Notice that machining error trajectories in Condition III significantly differ from those in Condition I and II, which is mostly attributable to the difference in the tilt angle of the workpiece, $\varphi$. It clearly shows that, in the five-axis machining, the effect of kinematic errors on the machine's positioning error may significantly differ depending on the location, the orientation, and the geometry of the workpiece. In all the conditions tested in case studies, simulated machining errors well match with measured ones. Even in Condition I, where circularity errors are as small as 7.1 9.3 $\mu \mathrm{m}$, simulated trajectories still show a good match with measured trajectories. This indicates that the machine's kinematic errors have notable effect on the machining geometric accuracy even under such a condition.

\subsection{Compensation of machining errors}

In order to justify its effectiveness, the presented error compensation scheme is demonstrated to the case study under Condition III. Cutting experiments for error compensation is carried out using the same cutting conditions as in the machining under Condition III. Figure 10 shows the compensated machining error trajectories for both bottom and top surfaces of the machined cone frustum. Compared to Fig. 9, it is seen that by applying the error compensation scheme, circularity errors are reduced from $17.3 \mu \mathrm{m}$ to $10.1 \mu \mathrm{m}$ at bottom and $19.5 \mu \mathrm{m}$ to $11 \mu \mathrm{m}$ at top surfaces of the machined cone frustum, and hence machining accuracy is improved significantly.

\section{Conclusions}

This paper presents a scheme to predict and evaluate the machining accuracy of a five-axis machining center with its kinematic errors. Three kinematic errors associated with linear axes and eight kinematic errors associated with rotary axes of the machining center are considered and identified practically by a DBB method. By using an error model with the kinematic errors, threedimensional interference of the tool and the workpiece is calculated along the tool path to simulate the machining geometric error under the influence of the machine's kinematic errors. As an application example, the present scheme is applied to the machining of a cone frustum, which is described in the standard NAS979 and is widely accepted in the machine tool industry as a machining performance test for five-axis machines. Case studies with cutting experiments on the target five-axis machining center verify the effectiveness of the prediction of machining errors in five-axis machining. Further, in order to enhance machining accuracy, an error compensation for tool position and orientation is presented. Experiments for error compensation show a significant 
improvement in machining accuracy in terms of reduction of circularity errors of the machined surface.

\section{References}

[1] ISO 10791 series, Test conditions for machining centers, ISO

[2] ISO 230-7: Test code for machine tools - Part 7: Geometric accuracy of axes of rotation, 2005

[3] NAS 979: Uniform cutting test - NAS series, metal cutting equipments, 1969

[4] Lin Y, Shen Y, Modeling of five-axis machine tool metrology models using the matrix summation approach, International Journal of Advanced Manufacturing Technology, 2003; $21 ; 243-248$

[5] Srivastava AK, Veldhuis SC, Elbestawi MA, Modeling geometric and thermal errors in a fiveaxis CNC machine tool, International Journal of Machine Tools \& Manufacture, 1994; 35(9); $1321-1337$

[6] Soons JA, Theuws FC, Schllekens PH, Modeling the errors of multi-axis machines: a general methodology, Precision Engineering, 1992; 14(1); 5-19

[7] Suh SH, Lee ES, Jung SY, Error modeling and measurement for the rotary table of five-axis machine tools, International Journal of Advanced Manufacturing Technology, 1998; 14; 656663

[8] Mayer JRR, Mir YA, Fortin C, Calibration of a five-axis machine tool for position independent geometric error parameters using a telescopic magnetic ball bar, Proceedings of the $33^{\text {rd }}$ International MATADOR Conference, 1999. pp 275-280

[9] Abbaszaheh-Mir Y, Mayer JRR, Clotier G, Fortin C, Theory and simulation for the identification of the link geometric errors for a five-axis machine tool using a telescoping magnetic ball-bar, International Journal of Production Research, , 2002; 40(18); 4781-4797

[10] Sakamoto S, Inasaki I, Identification of alignment errors in five-axis machining centers, Transactions of JSME, 1994; 60 (C-575); 2475-2483 (in Japanese)

[11] Kakino Y, Ihara T, Sato H, Otsubo H, A Study on the motion accuracy of NC machine tools (7th report) - measurement of motion accuracy of 5-axis machine by DBB tests, Journal of Japan Society for Precision Engineering, 1994; 60 (5); 718-723 (in Japanese)

[12] Tsutsumi M, Saito A, Identification of angular and positional deviations inherent to 5-axis machining centers with a tilting-rotary table by simultaneous four-axis control movement, International Journal of Machine Tools and Manufacture, 2004; 44; 1333-1342 
[13] Tsutsumi M and Saito A, Identification and compensation of systematic deviations particular to 5-axis machining centers, International Journal of Machine Tools \& Manufacture, 2003; 43; $771-780$

[14] Lei WT, Hsu YY, Accuracy test of five-axis CNC machine tool with 3D probe-ball. Part II: errors estimation, International Journal of Machine Tools and Manufacture, 2002; 42; 1163 1170

[15] Bringmann B, Knapp W, Model-based 'Chase-the-Ball' calibration of a 5-axis machining center, Annals of the CIRP, 2006; 55(1); 531-534

[16] Matsushita T, The accuracy of cone frustum machined by five-axis machining center with titling table, Proceedings of 2007 JSPE Spring Annual Conference, 2007. pp 187-188 (in Japanese)

[17] Yumiza D, Yoshinobu S, Tsutsumi M, Utsumi K, Ihara Y, Measurement method for motion accuracies of 5 -axis machining centers $-2^{\text {nd }}$ report, influence of geometric deviations on finished cone frustums - Proceedings. of 2007 JSPE Spring Annual Conference, 2007. pp 191-192 (in Japanese)

[18] JIS B 6310: Industrial automation systems and integration - Numerical control of machines coordinate system and motion nomenclature, Japanese Standard Association, 2003

[19] Inasaki I, Kishinami K, Sakamoto S, Sugimura N, Takeuchi Y, Tanaka F, Shaper generation theory of machine tools - its basis and applications, Yokendo, Tokyo, 1997. pp. 95-103 (in Japanese)

[20] Slocum AH, Precision Machine Design, Prentice-Hall, Englewood Cliffs, 1992

[21] Anderson RO, Detecting and eliminating collisions in NC machining, Computer-Aided Design, 1978; 10(4); 255-268

[22] Walstra WH, Bronsvoort WF, Vergeest JSM, Interactive simulation of robot milling for rapid shape prototyping, Computer Graphics, 1994; 18(6); 861-871

[23] Veldhuis SC, Elbestawi MA, A strategy for the compensations of the errors in five-axis machining, Annals of the CIRP, 1995; 44(1); 373-377

[24] Mahbubur RMD, Heikkala J, Lappalainen K, Karjalainen JA, Positioning accuracy improvement in five-axis milling by postprocessing, International Journal of Machine Tools \& Manufacture, 1997; 37(2); 223-236

[25] Hsu YY and Wang SS, A new compensation method for geometry errors of five-axis machine tools, International Journal of Machine Tools and Manufacture, 2007; 47(2); 352-360 


\section{List of Table Captions}

Table 1 Identified kinematic errors by the DBB tests

Table 2 Machining conditions of the tilted cone frustum used in simulation and experiment

Table 3 Summary of circularity and concentricity errors of the machined cone frustum in finishing under Condition I, II and III 


\section{List of Figure Captions}

Figure 1 Configuration of a five-axis machine tool of tilting rotary table type

Figure 2 Coordinate systems for the kinematic chain of a five-axis machining center with a tilting rotary table [12]

Figure 3 Machining configuration for the tilted cone frustum

Figure 4 Nominal geometry of bottom and top surfaces of the cone frustum and their normal vectors

Figure 5 Algorithm for the computation of the machining geometric errors

Figure 6 DBB measurement patterns used to identify kinematic errors

Figure 7 Simulated and measured machining error trajectories of the machined cone frustum surface in finishing under Condition I

Figure 8 Simulated and measured machining error trajectories of the machined cone frustum surface in finishing under Condition II

Figure 9 Simulated and measured machining error trajectories of the machined cone frustum surface in finishing under Condition III

Figure 10 Compensated machining error trajectories of the machined cone frustum in finishing under Condition III 
Table 1 Identified kinematic errors by the DBB tests

\begin{tabular}{llll}
\hline \multicolumn{2}{l}{8 kinematic errors with rotary axes } & \multicolumn{2}{c}{ 3 squareness errors with linear axes } \\
\hline Error parameter & Value & Error parameter & Value \\
\hline$\alpha_{A Y}$ & $+0.0001^{\circ}$ & $\gamma_{Y X}$ & $-0.0034^{\circ}$ \\
$\beta_{A Y}$ & $-0.0071^{\circ}$ & $\alpha_{Z Y}$ & $+0.0054^{\circ}$ \\
$\gamma_{A Y}$ & $-0.0081^{\circ}$ & $\beta_{Z X}$ & $+0.0037^{\circ}$ \\
$\beta_{C A}$ & $+0.006^{\circ}$ & & \\
$\delta x_{A Y}$ & $+0.10 \mu \mathrm{m}$ & & \\
$\delta y_{A Y}$ & $-4.0 \mu \mathrm{m}$ & & \\
$\delta z_{A Y}$ & $+2.8 \mu \mathrm{m}$ & & \\
$\delta y_{C A}$ & $+3.4 \mu \mathrm{m}$ & & \\
\hline
\end{tabular}


Table 2 Machining conditions of the tilted cone frustum used in simulation and experiments

\begin{tabular}{llll}
\hline $\begin{array}{l}\text { Machining parameter of the } \\
\text { cone frustum }\end{array}$ & Condition I & Condition II & Condition III \\
\hline $\begin{array}{l}\text { Diameter of the cone frustum } \\
\text { machining tool path, } \phi D(\mathrm{~mm})\end{array}$ & 194 & 210 & 206 \\
Tilt angle, $\varphi\left(^{\circ}\right)$ & 7.5 & 15 & 75 \\
Taper angle, $\theta\left(^{\circ}\right)$ & 15 & 30 & 30 \\
Center location of workpiece & $(0,-100,43)$ & $(0,-100,53)$ & $(0,-103,93)$ \\
$\left(\mathrm{C}_{\mathrm{x}}, \mathrm{C}_{\mathrm{y}}, \mathrm{C}_{\mathrm{z}}\right)(\mathrm{mm})$ & & & \\
\hline
\end{tabular}


Table 3 Summary of circularity and concentricity errors of the machined cone frustum in finishing under Condition I, II and III

\begin{tabular}{lllllll}
\hline & \multicolumn{2}{l}{ Condition I } & \multicolumn{2}{l}{ Condition II } & \multicolumn{2}{c}{ Condition III } \\
\cline { 2 - 7 } & Simulated & Measured & Simulated & Measured & Simulated & Measured \\
\hline $\begin{array}{l}\text { Circularity error at } \\
\text { bottom }(\mu \mathrm{m})\end{array}$ & 6.3 & 7.1 & 7.2 & 9.3 & 13.3 & 17.3 \\
$\begin{array}{l}\text { Circularity error at } \\
\text { top }(\mu \mathrm{m})\end{array}$ & 5.8 & 9.3 & 6.7 & 8.8 & 13.0 & 19.5 \\
$\begin{array}{l}\text { Concentricity error } \\
(\mu \mathrm{m})\end{array}$ & 0.85 & 5.4 & 1.0 & 0.6 & 3.8 & 1.9 \\
\hline
\end{tabular}




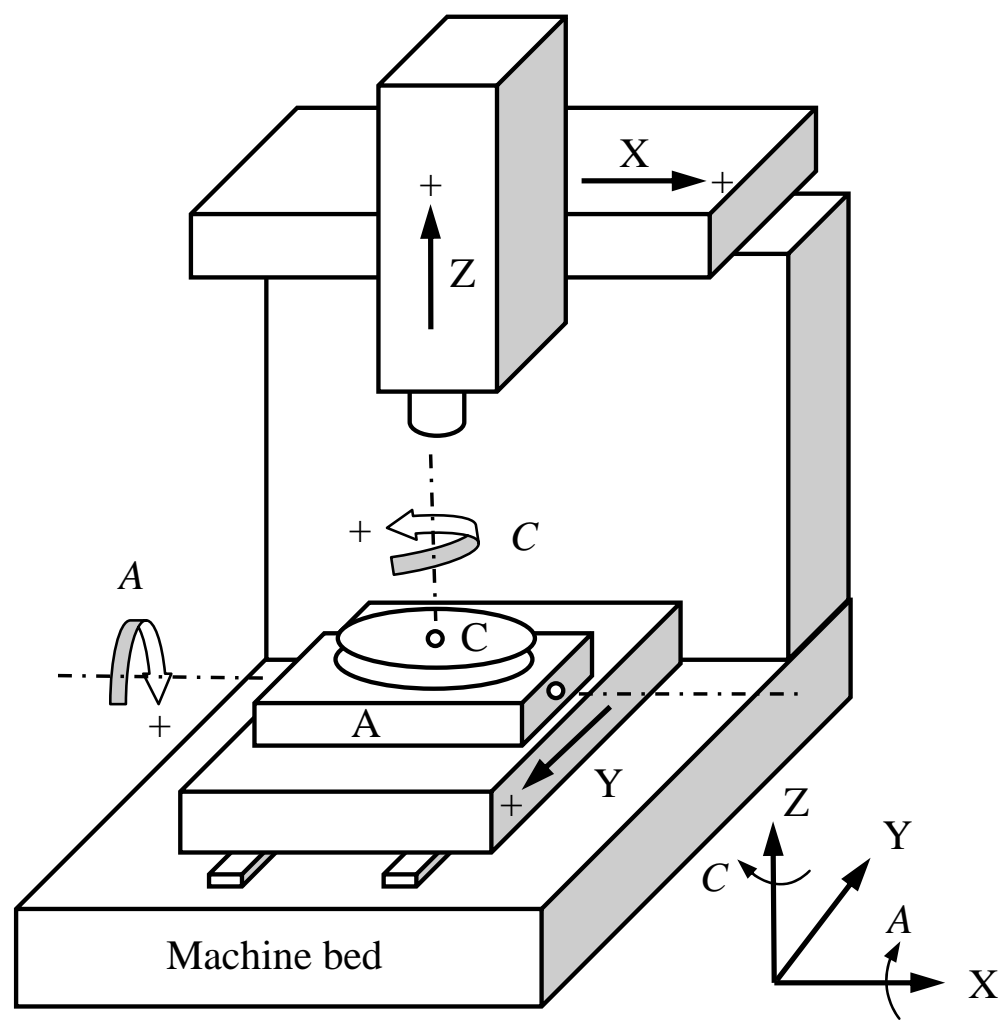

Figure 1 Configuration of a five-axis machine tool of tilting rotary table type 


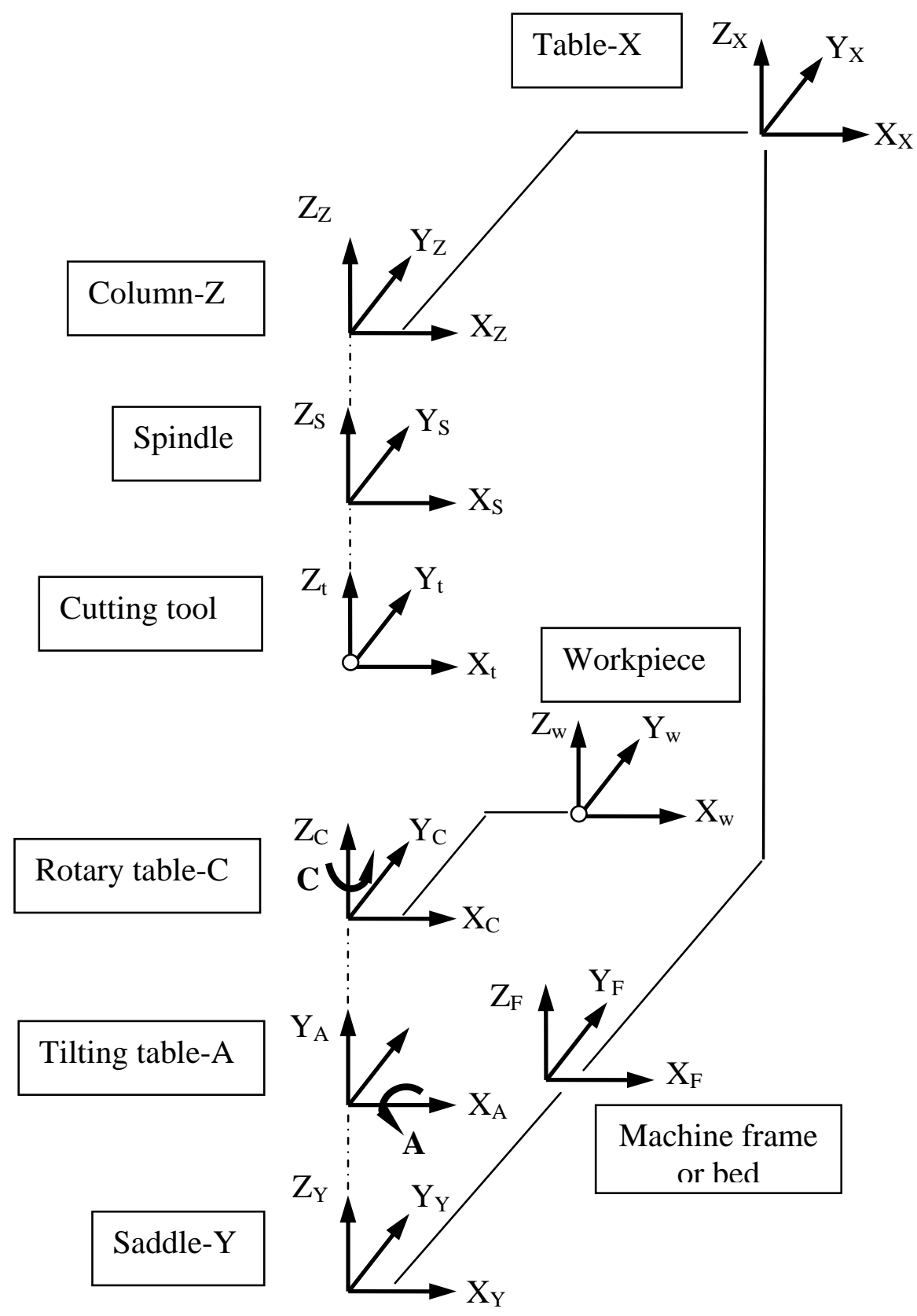

Figure 2 Coordinate systems for the kinematic chain of a five-axis machining center with a tilting rotary table [12] 


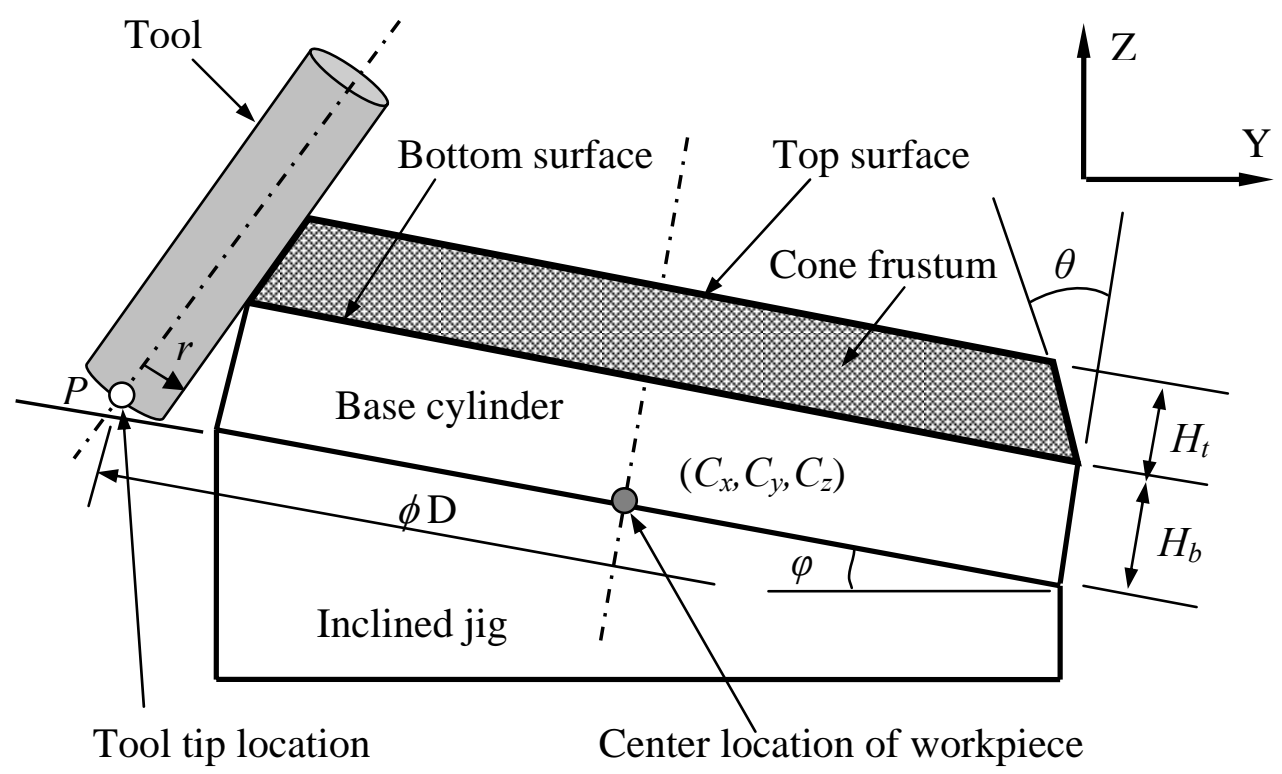

Figure 3 Machining configuration for the tilted cone frustum 


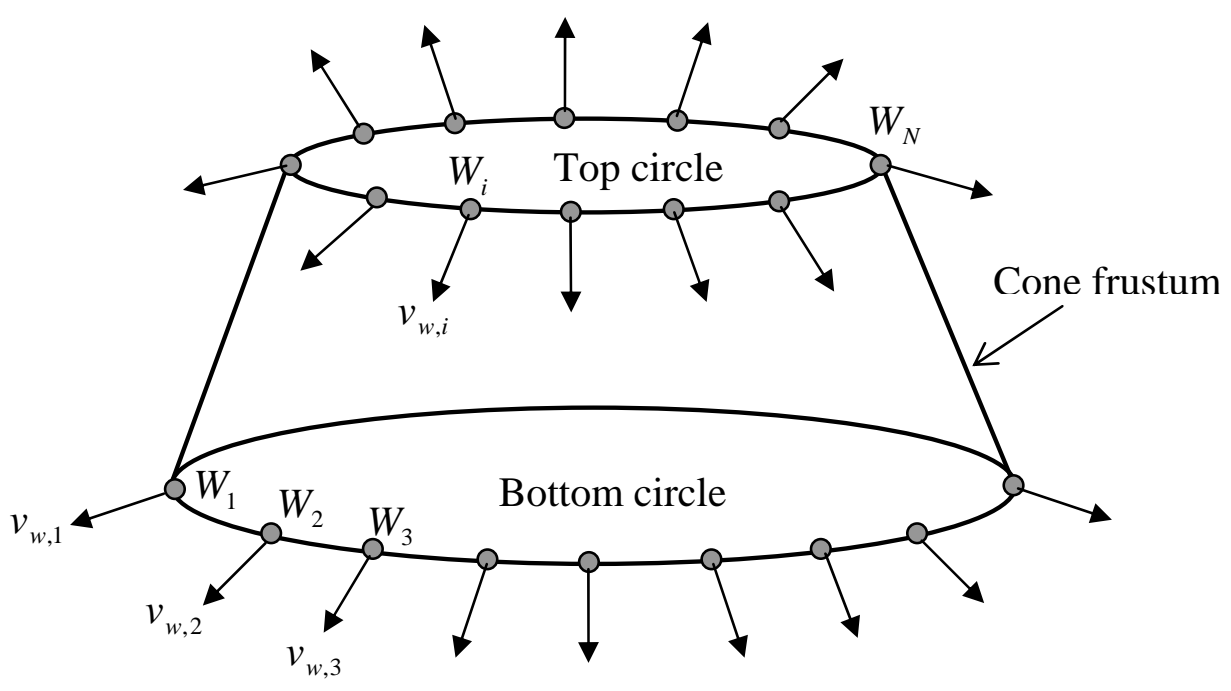

Figure 4 Nominal geometry of bottom and top surfaces of the cone frustum and their normal vectors 


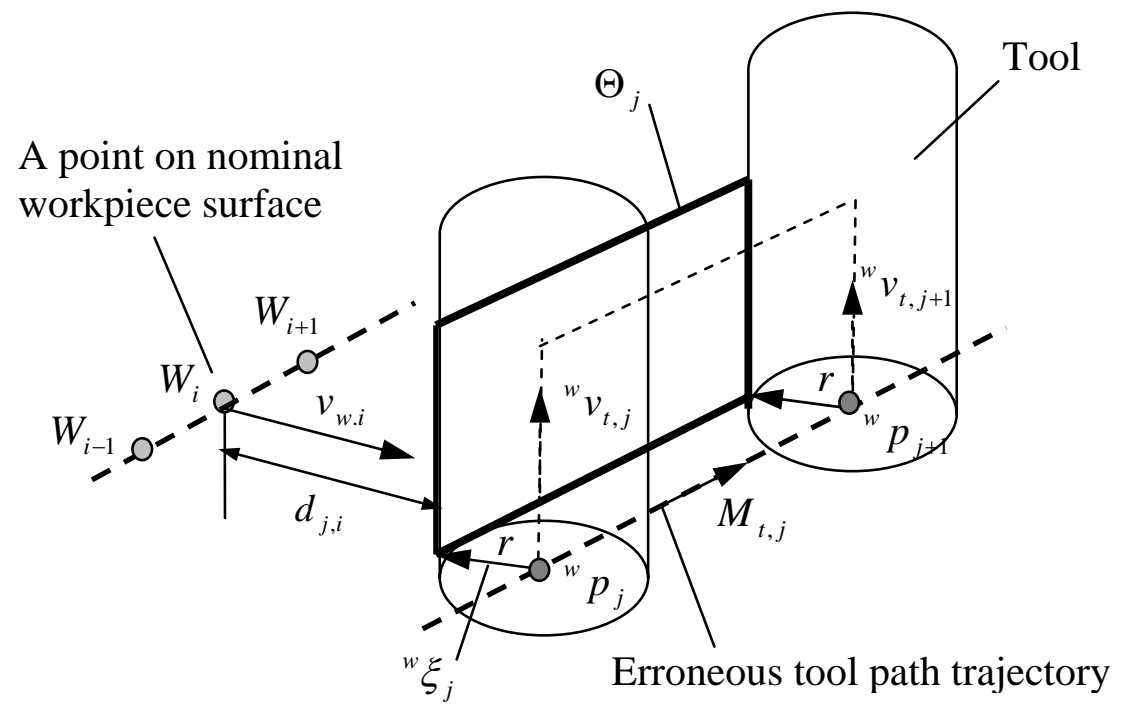

Figure 5 Algorithm for the computation of the machining geometric errors 

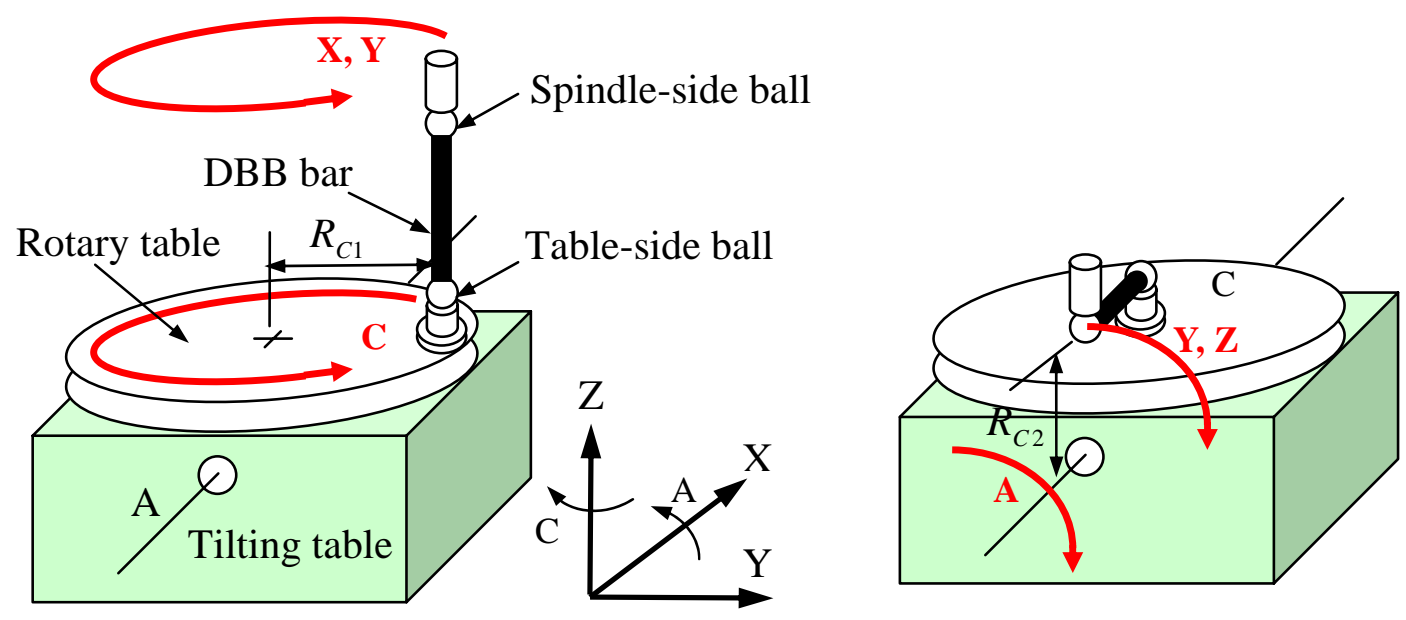

(a) DBB measurement pattern 1

(b) DBB measurement pattern 2

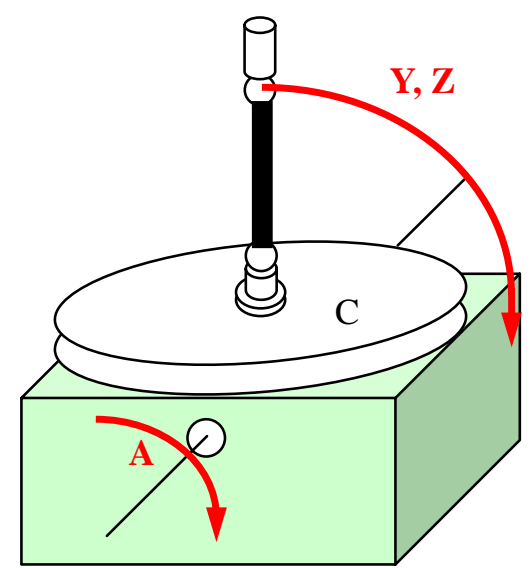

(c) DBB measurement pattern 3

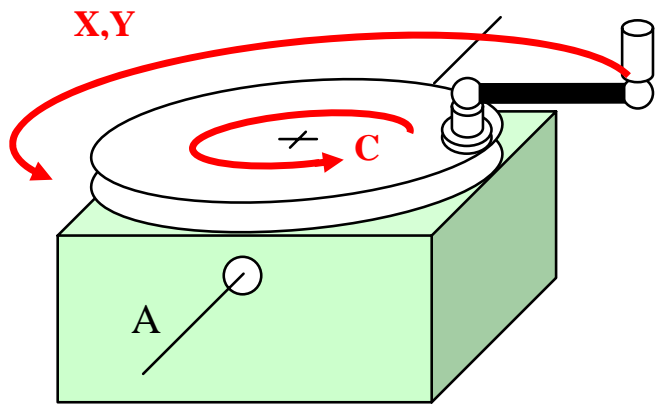

(d) DBB measurement pattern 4

Figure 6 DBB measurement patterns used to identify kinematic errors 


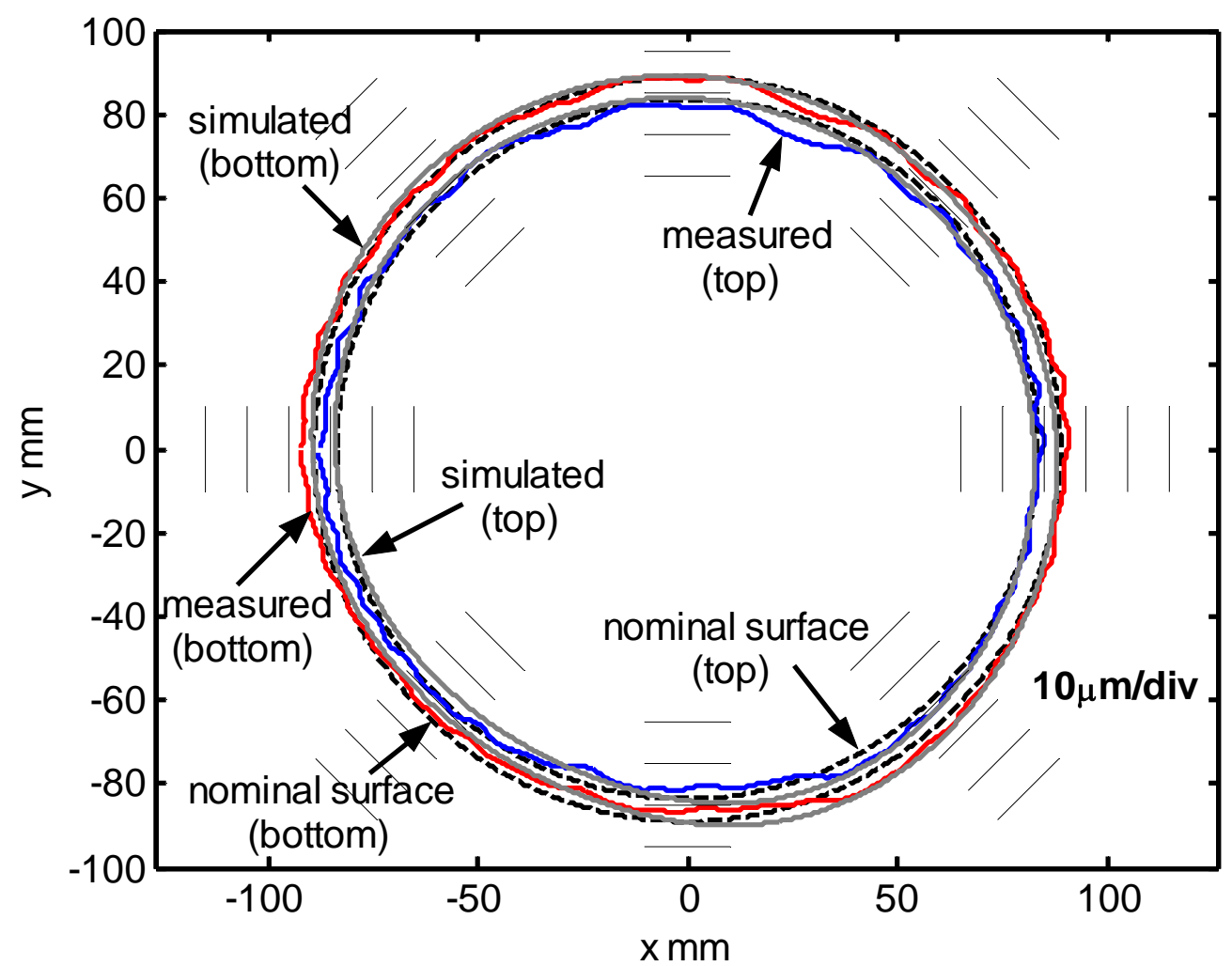

Figure 7 Simulated and measured machining error trajectories of the machined cone frustum surface in finishing under Condition I 


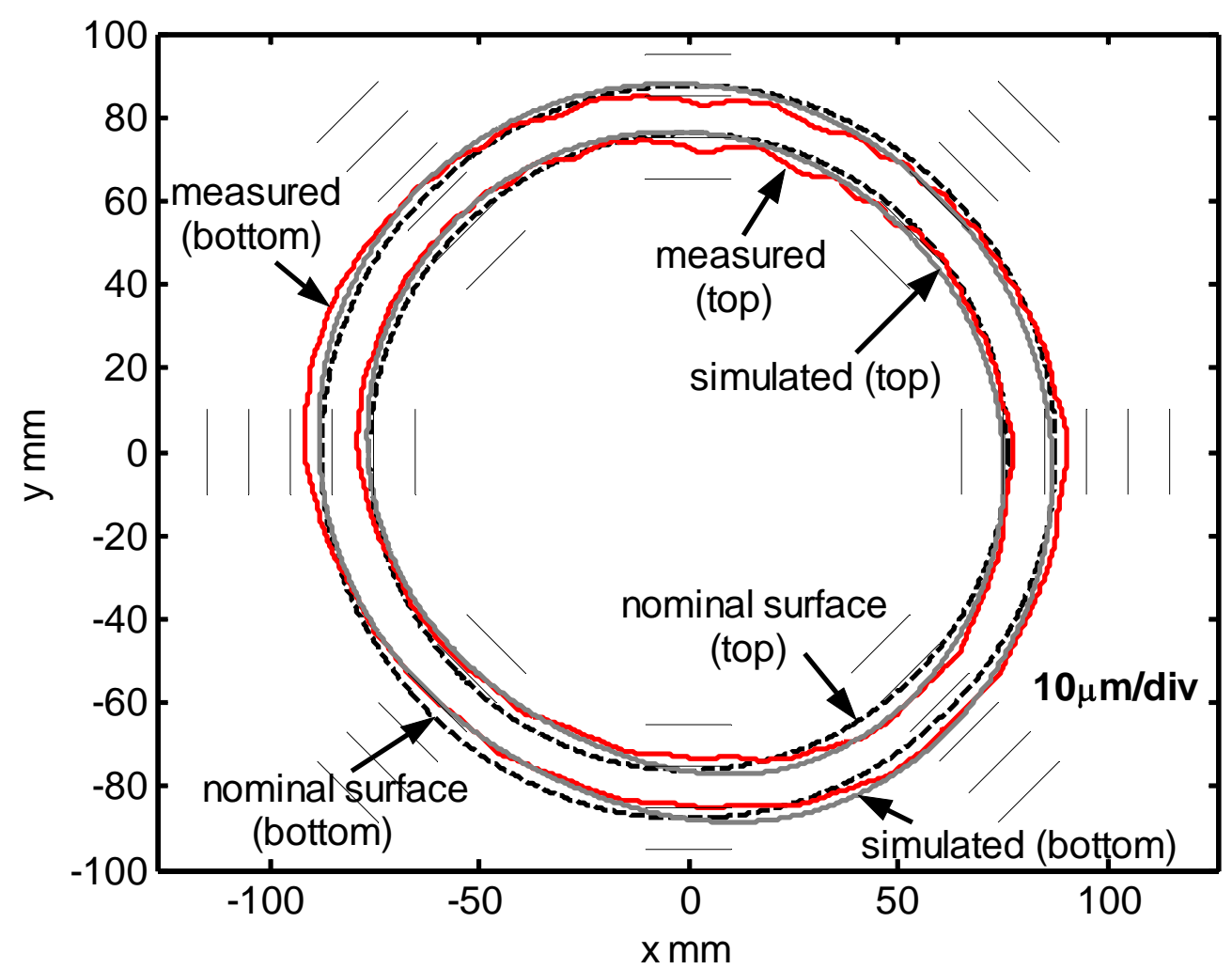

Figure 8 Simulated and measured machining error trajectories of the machined cone frustum surface in finishing under Condition II 


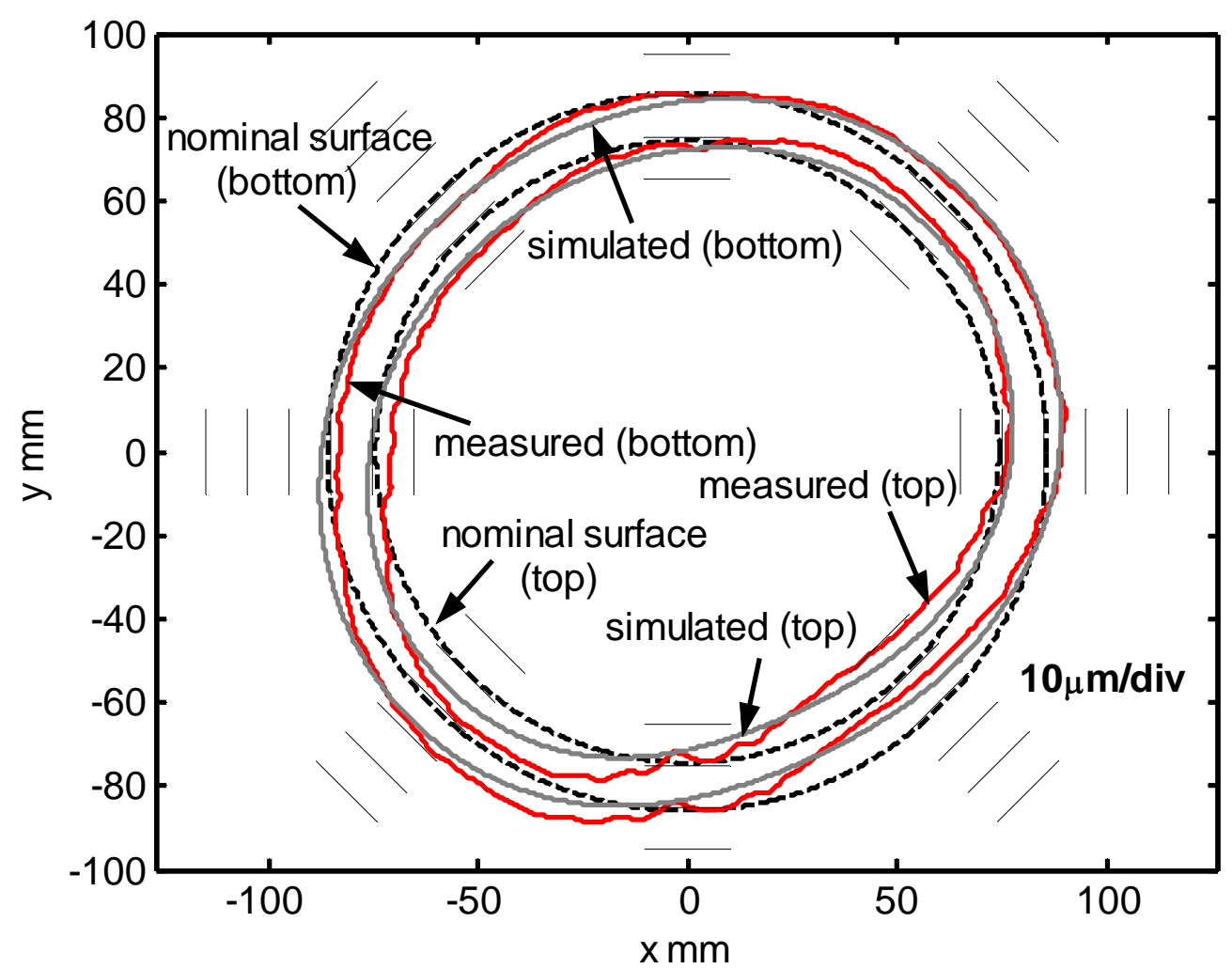

Figure 9 Simulated and measured machining error trajectories of the machined cone frustum surface in finishing under Condition III 


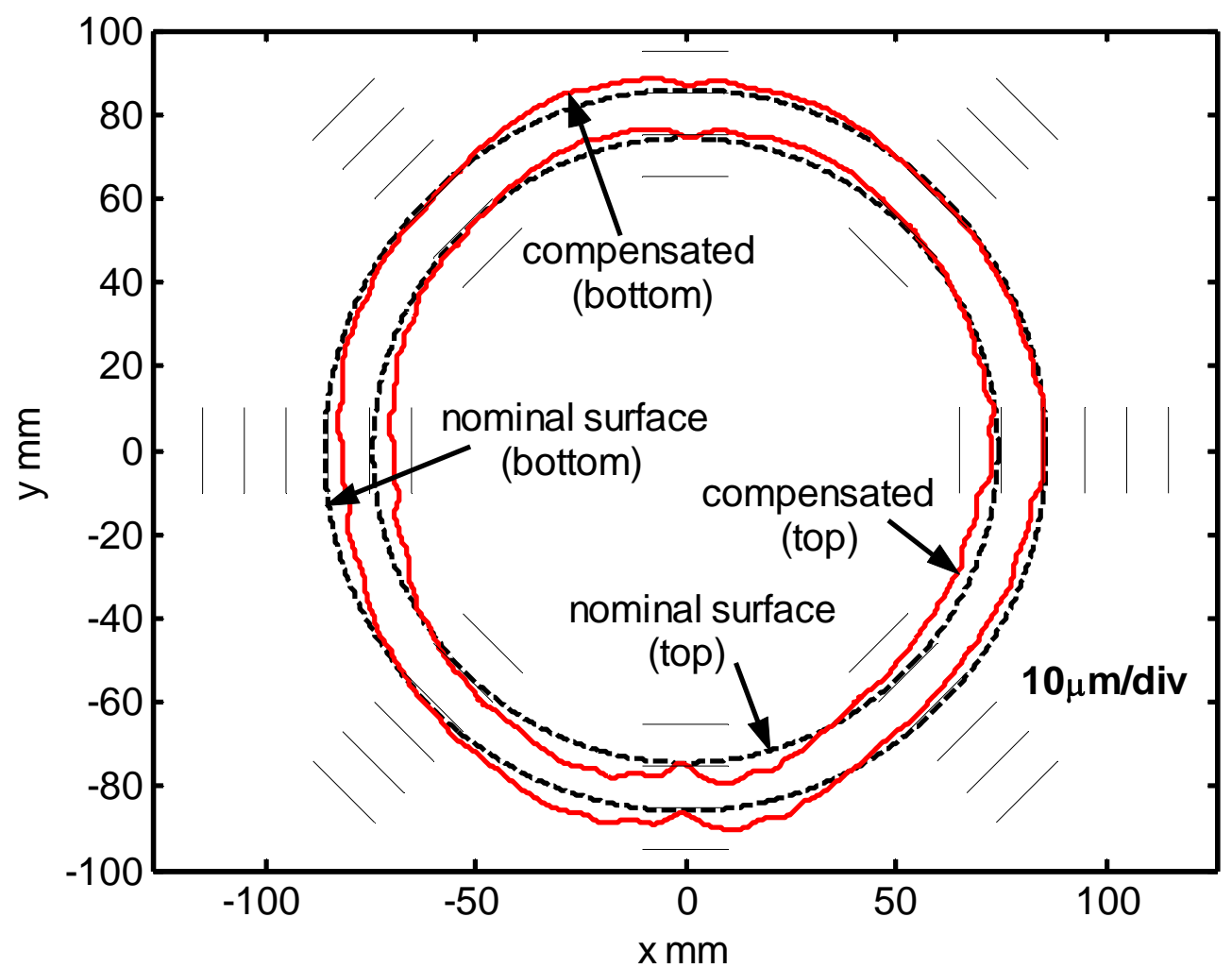

Figure 10 Compensated machining error trajectories of the machined cone frustum in finishing under Condition III 